\title{
EDITORIAL
}

\section{"Gemeinsam geht es besser"}

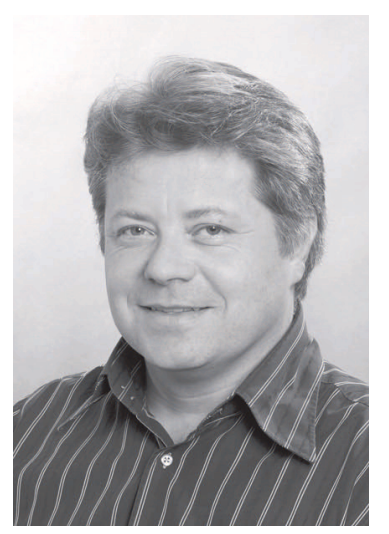

Messen wandeln sich. Denn sie richten sich nach den Erfordernissen der Märkte, die sie abbilden.

So hat sich auch die ILMAC gewandelt. 1959 fand sie erstmals als 'Internationale Fachmesse für Laboratoriums- und Messtechnik und Automatik in der Chemie' statt. 1968 wurde die 'ilmac' um die Verfahrenstechnik erweitert. 2002 kam dann der Versuch, die Themenbereiche Forschung und Entwicklung sowie Verfahrens- und Umwelttechnik in eigenständigen Messen zu platzieren. Ein Erfordernis des Marktes - so schien es. Und selbiger fordert nun wieder eine gemeinsame Plattform für alle industriellen Anwendungen der Verfahrenstechnik - sei es in der Forschung und Entwicklung, in der Pilotierung und im Engineering oder in der Produktion und Entsorgung.

Eine Konstante in diesem Wandel ist die Schweizerische Chemische Gesellschaft: Sie war und ist uns eine wichtige Partnerin. Die SCG hat als Begründerin die Messe über Jahre hinweg mit uns gemeinsam aufgebaut und mitgetragen. Sie verfügt über das Know-how auf den Gebieten der Chemie und verwandter Wissenschaften, um eine für die durch sie vertretenen Chemiker interessante Plattform zu gestalten.

Jedoch hat sich die Art und Weise der Zusammenarbeit im Laufe der Jahre verändert. Organisierte die SCG bisher ganztägige Tagungen und Symposien, ab 1996 gar einen parallel zur Messe stattfindenden Kongress, steht jetzt wieder die 'Vereinbarkeit' von Vortrags- und Messebesuch im Vordergrund. Des Weiteren ist die Themenauswahl anwendungsorientiert, Wissenschaftler aus Firmen und von Hochschulen berichten praxisnah über neueste Entwicklungen und Best Practices. Nach den Vorträgen geht die Wissensvermittlung weiter: bei den über 450 Ausstellern vor Ort.

Die Schweizerische Chemische Gesellschaft und die Messe Schweiz laden Sie herzlich ein, sich über die aktuellen Anwendungen und neuesten Produkte für die Pharma-, Chemie- und Biotechnologiebranchen zu informieren.

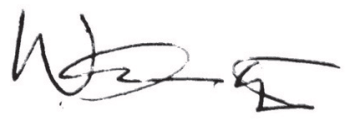

Christian Wenger

Leiter Industriefachmessen

Messe Schweiz 